\title{
CHAOS IN THE OUTER SOLAR SYSTEM
}

\author{
D. Babich ${ }^{1}$
}

\begin{abstract}
We study the effect of a perturber on the energy and angular momentum of a test particle on a highly eccentric orbit. We find that both the energy and angular momentum of the test particle are adiabatic invariants when the perturber is on a circular orbit with radius much smaller than the periaps of the test particle. We derive analytical expression for the energy change, or kick, of the test particle with every periaps passage. Using this kick function we formulate mapping for the highly eccentric test particle. We also analyze the stability of resonances and find conditions for asymmetric libration to occur. The resonance widths for highly eccentric orbits are calculated and the criterion for the onset of global chaos is determined.
\end{abstract}

\section{Introduction}

Objects in the outer Solar System, significantly beyond the eight planets, are widely believed to be remnants of the early history of the Solar System. The dynamical time in the outer Solar System is too long, and densities are thought to be too low, for these objects to have formed at their present locations. The currently accepted hypothesis is that these objects formed much closer in the Solar System and were perturbed into the outer Solar System by the giant planets. These objects and their orbits provide us with clues about the formation process of the Solar System, as well as, its subsequent dynamical evolution. Interaction with the planets tends to increase the object's energy while approximately maintaining its distance of periapse, therefore placing it on a highly eccentric orbit. In this paper we study the dynamical interaction of particles on these highly eccentric orbits with the planets.

We derive a mapping of a test particle on an highly eccentric orbit which evolves due to the energy kicks by a perturber on a circular orbit. The energy and phase shifts per periapse passage, are derived in the approximation that the test particle

1 California Institute of Technology, Theoretical Astrophysics, MC 130-33, Pasadena, CA 91125, USA; e-mail: babich@tapir.caltech.edu 
is on a parabolic orbit. This analysis is a generalization of the work of (Pan \& Sari 2004), who considered test particles whose distance of periapse is $9 / 8$ of the semi-major axis of the circular perturber, to test particles with larger periapses. This limit of large periapse allows as to derive analytic results and is relevant for objects like Sedna (Brown et al. 2004), whose periapse at $76 \mathrm{AU}$ is well outside the reach of planets or comets currently residing in the Oort Cloud.

\section{Kick Functions}

The potential produced by the perturber can be integrated over the unperturbed path of the test particle to calculate, to first order in $\mu$, the change in the action due to the perturber

$$
\Delta S=-\int_{-\infty}^{+\infty} \mathrm{d} t V(t) .
$$

Then by integrating Hamilton's equations we find that the total change in the angular momentum, the conjugate momentum to the phase angle, is

$$
\Delta J=-\frac{\partial}{\partial \phi} \Delta S,
$$

and that a net phase shift occurs during the period of the test particle

$$
\Delta \phi=\frac{\partial}{\partial J} \Delta S .
$$

The angular momentum is related to the distance of periapse as $J=\sqrt{2 r_{p}}$. The. change in angular momentum can be related to the change in the energy via the exact conservation of the Jacobi constant in the circular three-body problem, $\Delta E=\Delta J / \bar{a}^{3 / 2}$.

The potential can be expanded in a Legendre series, in the physically relevant case of $r_{p}>\bar{a}$, as

$$
V=-\frac{\mu}{r} \sum_{n=2}\left(\frac{\bar{a}}{r}\right)^{n} P_{n}(\cos \lambda) .
$$

We have restricted the index in the Legendre series to $n \geq 2$ because the monopole term $(n=0)$ does not produce a force on the test particle and the dipole term $(n=1)$ in the expansion of the direct piece is cancelled by the indirect piece of the perturbation.

Evaluating the integral using the residue theorem and multiple integrationby-parts, we find that the change in the action, expressed in terms of a cosine series, is

$$
\begin{aligned}
\Delta S(\phi, \beta) \simeq & \frac{1}{4} \mu \sqrt{2 \pi \bar{a}}(6 \beta)^{-1 / 6} e^{-2 \beta} \cos (\phi)\left[1-\frac{1}{288 \beta}+\mathcal{O}\left(\beta^{-2}\right)\right] \\
& +4 \mu \sqrt{6 \pi \bar{a} \beta} e^{-4 \beta} \cos (2 \phi)\left[1+\frac{67}{144 \beta}+\mathcal{O}\left(\beta^{-2}\right)\right]
\end{aligned}
$$


which leads to the following expression for the energy change per orbit

$$
\begin{aligned}
\Delta E(\phi, \beta) \simeq & \frac{\mu}{4 \bar{a}} \sqrt{2 \pi}(6 \beta)^{-1 / 6} e^{-2 \beta} \sin (\phi)\left[1-\frac{1}{288 \beta}+\mathcal{O}\left(\beta^{-2}\right)\right] \\
& +\frac{8 \mu}{\bar{a}} \sqrt{6 \pi \beta} e^{-4 \beta} \sin (2 \phi)\left[1+\frac{67}{144 \beta}+\mathcal{O}\left(\beta^{-2}\right)\right] .
\end{aligned}
$$

The change in action contains both higher order cosine harmonics, as well as, additional terms that scale as inverse powers of $\beta$ for each term in the cosine series. Equation (2.5) only displays the leading order terms. Since the coefficient for the $\cos (k \phi)$ term scales as $e^{-2 k \beta}$, these higher harmonic terms rapidly become irrelevant.

The energy kick function we derived can be used to define a mapping of the orbit of our test particle from one period to the next. This is a very efficient means of analyzing the dynamics of this problem because the impulse applied on the test particle is localized near periapse. While we have previously assumed the test particle was on a parabolic orbit in order to calculate the mapping coefficients, we will now treat the test particle as bound, but highly eccentric. Combing the energy and phase shifts calculated in the previous sections this mapping can be written as

$$
\begin{gathered}
\frac{1}{a_{n+1}}=\frac{1}{a_{n}}-2 \Delta E\left(\phi_{n}, \beta\right), \\
\phi_{n+1}=\phi_{n}+2 \pi\left(\frac{a_{n+1}}{\bar{a}}\right)^{3 / 2},
\end{gathered}
$$

where $\phi_{n}$ is defined modulo $2 \pi$. Here $a_{n}$ is the semi-major axis of the test particle during the $n^{\text {th }}$ iteration of the mapping.

Resonances in the three-body problem appear as fixed points in our mapping model. As discussed in (Pan \& Sari 2004), the standard labeling of the strength of a resonance by the number of conjunction per resonant cycle is meaningless in the highly eccentric limit because the impulse at periapse dominates the total force applied on the test particle. The 1: $N$ resonances described in (Pan \& Sari 2004) can be found by calculating phase angles where the energy kick vanishes; here $N$ is related to the semi-major axes of the particles as $N=(a / \bar{a})^{3 / 2}$. The energy kick function can be expressed as a sine series

$$
\Delta E(\phi, \beta)=\sum_{k=1}^{\infty} \Gamma_{k}(\beta) \sin (k \phi),
$$

where the series coefficients only depend on $\beta$. In Equation (2.6) we only gave expressions for $k=1,2$ but any term can be calculated using the method described in that section. Since the energy kick function series coefficients scale as $\Gamma_{k}(\beta) \propto$ $e^{-2 k \beta}$, the higher order terms become irrelevant quite rapidly.

In the large $\beta$ limit where a single perturbing term dominates the energy kick function, we can relate the mapping defined in the previous section to the standard 
map, a mathematical model used to study the properties of dynamical systems (Litchenberg \& Lieberman 1992). The standard map is defined as

$$
\begin{gathered}
J_{n+1}=J_{n}+K \sin \left(\phi_{n}\right), \\
\phi_{n+1}=\phi_{n}+J_{n+1} .
\end{gathered}
$$

In order to rewrite our mapping in this form, we define the variable $J_{n} \equiv$ $2 \pi\left(a_{n} / \bar{a}\right)^{3 / 2}$, to describe the phase shift per orbit. In the large $\beta$ limit only the $k=1$ term of Equation (2.9) is important. Using these approximations our mapping becomes

$$
\begin{gathered}
J_{n+1}=J_{n}+\frac{3 \pi}{2} \mu\left(\frac{a_{c}}{\bar{a}}\right)^{5 / 2} \frac{\sqrt{2 \pi}}{(6 \beta)^{1 / 6}} e^{-2 \beta} \sin \left(\phi_{n}\right)\left[1+\frac{1}{288 \beta}+\mathcal{O}\left(\beta^{-2}\right)\right] \\
\phi_{n+1}=\phi_{n}+J_{n+1} .
\end{gathered}
$$

Surface-of-section analyses have determined that the onset of chaos in the standard map occurs when $K_{c}=0.97$ (Litchenberg \& Lieberman 1992; Chirikov 1979). This criterion was determined numerically (Chirikov 1979) and is more precise than the heuristic resonance overlap criterion. This implies that for a given $r_{p}$ the semimajor axis of the test particle must be greater than the critical value

$$
a_{c}=\bar{a}\left(\frac{2 K_{c}}{3 \pi \sqrt{2 \pi}}\right)^{2 / 5}(6 \beta)^{1 / 15} \mu^{-2 / 5} e^{4 \beta / 5}\left[1-\frac{1}{720 \beta}+\mathcal{O}\left(\beta^{-2}\right)\right] .
$$

The functional dependence on $\mu$ and $\beta$ is identical to criterion derived from resonance overlap, only the numerical coefficient differs between the two criteria. The resonance overlap requirement predicts that chaos occurs at a semi-major axis nearly 3.5 times larger than the prediction of the standard map criterion. This result is consistent with the finding that the resonance overlap criterion generally indicates a transition to chaos at stronger perturber strength then is found using numerical methods. Within the approximation of our model we can conclude that Sedna is not on a chaotic orbit, although a fraction of the comets in the Oort Cloud are undergoing chaotic motion.

\section{References}

Brown, M.E., et al., 2004, ApJ, 617, 645

Chirikov, B.V., 1979, Phys. Repts., 52, 263

Lichtenberg, A.J., \& Lieberman, M.A., 1992, Regular and Chaotic Dynamics, $2^{\text {nd }}$ ed. (New York: Springer-Verlag)

Murray, C.D., \& Dermott, S.F., 1999, Solar System Dynamics (New York: Cambridge University Press)

Pan, M., \& Sari, R., 2004, AJ, 128, 1418 